ఠ

REVIEW

\title{
Resistance and the management of complicated skin and skin structure infections: the role of ceftobiprole
}

This article was published in the following Dove Press journal:

Therapeutics and Clinical Risk Management

25 September 2010

Number of times this article has been viewed

\author{
April Barbour ${ }^{\prime}$ \\ Hartmut Derendorf² \\ 'GlaxoSmithKline, King of Prussia, PA, \\ USA; ${ }^{2}$ Department of Pharmaceutics, \\ College of Pharmacy, University of \\ Florida, Gainesville, FL, USA
}

\begin{abstract}
Antimicrobial resistant bacteria are an increasing concern due to the resulting increase in morbidity, mortality, and health-care costs associated with the administration of inadequate or delayed antimicrobial therapy. The implications of inadequate antimicrobial therapy in complicated skin and skin structure infections (cSSSIs) have gained more attention recently, most likely due to the recent emergence of community-acquired methicillin resistant Staphylococcus aureus (MRSA) and the already high prevalence of MRSA in the nosocomial setting. Due to the continuous threat of resistance arising and the limitations of currently available agents for the treatment of cSSSIs, it is necessary to develop new antimicrobials for this indication. Ceftobiprole medocaril, the prodrug of ceftobiprole, is a parental investigational cephalosporin for the treatment of cSSSIs displaying a wide-spectrum of activity against both Gram-positive and Gram-negative species, including MRSA. Ceftobiprole displays noncomplex linear pharmacokinetics, is eliminated primarily by glomerular filtration, and distributes to extracellular fluid. Additionally, it has been shown that the extent of distribution to the site of action with regard to cSSSIs, ie, the extracellular space fluid of subcutaneous adipose tissue and skeletal muscle, is expected to be efficacious, as free concentrations meet efficacy targets for most pathogens. Similar to other beta-lactams, it displays an excellent safety and tolerability profile with the primary adverse events being dysgeusia in healthy volunteers, resulting from the conversion of the prodrug to the active, and nausea in patients. Ceftobiprole has demonstrated noninferiority in two large-scale pivotal studies comparing it to vancomycin, clinical cure rates $93.3 \%$ vs $93.5 \%$, respectively, or vancomycin plus ceftazidime, clinical cure rates $90.5 \%$ vs $90.2 \%$, respectively. Given the pharmacokinetic and pharmacodynamic properties, ceftobiprole is a promising new agent for the treatment of cSSSIs and has the potential to be used as a single agent for empiric treatment.
\end{abstract}

Keywords: cSSSIs, resistance, MRSA, cephalosporins

\section{Introduction}

Antimicrobial resistant bacteria are an increasing concern due to the resulting increase in morbidity, mortality, and health-care costs associated with the administration of inadequate or delayed antimicrobial therapy. ${ }^{1-9}$ Proper guidelines should be followed to efficiently manage skin and skin structure infections and avoid the over usage of available antibiotics, which could possibly facilitate resistance development. The management of skin and skin structure infections is dependent on several factors. One factor is whether the infection is complicated or uncomplicated, as this dictates the need for antibiotics. Complicated infections are defined as infections involving deep soft tissues and usually require antimicrobial therapy and possibly surgical
Correspondence: Hartmut Derendorf 1600 SW Archer Rd, Room P3-20, PO BOX 100494, Gainesville, FL 32610, USA

Tel +l 3522737856

Fax +l 3523923249

Email hartmut@cop.ufl.edu 
intervention. Infections occurring in patients with significant comorbidities, eg, diabetes or eczema, or in certain anatomical locations, eg, perianal, may also be considered complicated. ${ }^{10-13}$ Uncomplicated infections, however, are more superficial and less serious, eg, impetigo, cellulitis, and folliculitis. ${ }^{10,12,13}$ Typically, uncomplicated skin and soft tissue infections are effectively managed with incision and drainage. ${ }^{14,15}$ The management of complicated infections is more complex and it is recommend that in addition to possible incision and drainage, the following be performed in order to optimize antimicrobial therapy; a detailed history, examination of the lesions as part of a physical exam, and administration of empiric antimicrobial therapy with adjustment after microbiological identification of the pathogen(s). ${ }^{16}$ These guidelines attempt to provide as much information as possible to health care providers so that optimal therapy can be started and treatment may be adjusted as additional information becomes available.

The selection of an empiric antibiotic with activity against the causative pathogen(s) is nevertheless, difficult due to resistance and the wide-range of possible pathogens. For example, Staphylococcus aureus and Pseudomonas aeruginosa respectively, account for approximately $45 \%$ and $11 \%$ of skin and soft tissue infections in North America. ${ }^{17}$ Despite these difficulties, selection of an appropriate antibiotic in a timely fashion is imperative due to the negative outcomes associated with inadequate antimicrobial therapy (IAT). The impact of IAT has been extensively studied in pneumonia and bloodstream infections due to the more severe clinical outcomes associated with these infections, ie, higher mortality rates. ${ }^{18}$ While there are some previous data that suggest that IAT results in negative outcomes in skin and soft tissue infections, ${ }^{7}$ the implications of IAT in complicated skin and skin structure infections (cSSSIs), including surgical site infections, have gained more attention recently. ${ }^{3,4,8}$

The high prevalence of methicillin resistant $S$. aureus in the nosocomial setting, or hospital-acquired MRSA (HA-MRSA), and the emergence of community-acquired methicillin-resistant $S$. aureus (CA-MRSA) ${ }^{19-21}$ is certainly a cause for concern and numerous studies have been conducted to evaluate the impact of CA-MRSA in particular. Studies have examined whether or not there has been a correlation between physician visits and the emergence of CA-MRSA. The literature on the effect of CA-MRSA on overall ambulatory visits is controversial and therefore difficult to interpret. However, numerous studies do in fact show a correlation between the emergence of CA-MRSA and an increase in emergency department visits and/or visit rates due to skin and soft tissue infections. ${ }^{22-24}$ Subsequently, there may also be an increase in prescriptions for antibiotics with activity against CA-MRSA. ${ }^{22,25}$ However, this literature is also controversial. ${ }^{23,24}$ Despite contradictory reports, it can be definitively stated that with the emergence and prevalence of resistant species, particularly resistance in Staphylococcus species (Figure 1), ${ }^{12}$ and the negative impacts of IAT, there is a need to develop new antimicrobial therapeutic options.

\section{Antimicrobial therapies for the treatment of cSSSIs}

When selecting empiric therapeutic options for the treatment of cSSSIs, it is necessary to consider regional resistance patterns to make an appropriate treatment recommendation. It has been recommended that for patients with skin and soft tissue infections (SSTIs) in regions where CA-MRSA prevalence is greater than $10 \%-15 \%$, alternatives to the commonly prescribed beta-lactams should be used. ${ }^{25,26}$ Additionally, it has been suggested that due to resistance, ciprofloxacin, levofloxacin, and clindamycin, are no longer viable empirical treatment options. ${ }^{27}$ Vancomycin has long been the standard empirical therapy for patients with serious cSSSIs, as it displays good activity against MRSA and other Gram-positive organisms commonly implicated in these infections. However, resistance to vancomycin is seen in Enterococcus species, $>10 \%,{ }^{17,28}$ and in a few case reports with $S$. aureus. ${ }^{29-31}$ In view of the problem of resistance, several new therapeutic options have been recently developed. However, many of these new treatments have serious limitations

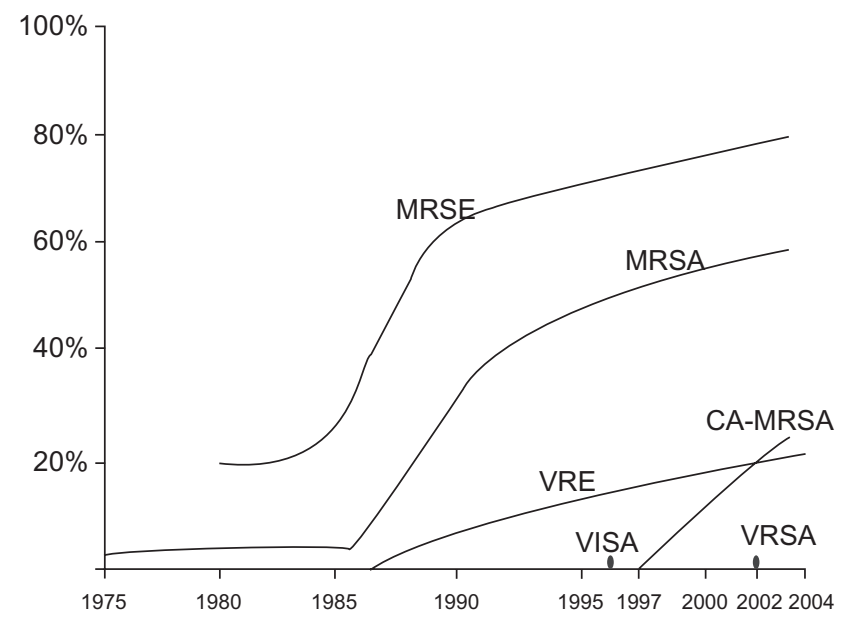

Figure I Trends in resistance among Gram-positive pathogens (1975-2004). MRSE: methicillin-resistant S. epidermidis. VRE: vancomycin-resistant Enterococcus. VISA: vancomycin-intermediate $S$. aureus. VRSA: vancomycin-resistant $S$. aureus. Reprinted with permission from Lee SY, Kuti JL, Nicolau DP. Antimicrobial management of complicated skin and skin structure infections in the era of emerging resistance. Surg Infect (Larchmt). 2005;6:283-295. ${ }^{12}$ Copyright (C) 2005 Mary Ann Liebert, Inc. publishers. 
including a narrow activity profile, eg, Gram-positive organisms only, and/or serious side effects.

Quinupristin-dalfopristin has been approved for the treatment of bacteremia due to vancomycin-resistant Enterococcus faecium and cSSSIs due to methicillinsusceptible $S$. aureus (MSSA) and Streptococcus pyogenes. ${ }^{32}$ However, it is not active against vancomycinresistant Enterococcus faecalis. ${ }^{32}$ Additionally, it is not indicated in infections against MRSA, although it has been suggested to be an acceptable treatment option ${ }^{33,34}$ and displays an in vitro $\mathrm{MIC}_{90} \leq 1 \mathrm{mg} / \mathrm{L} .{ }^{35-37}$ Finally, quinupristin-dalfopristin also bears a significant probability of myalgia as a side effect and requires a central venous catheter for administration, which limits its use. ${ }^{34,38}$ Linezolid is another available agent for the treatment of Gram-positive infections, including MRSA. Like vancomycin, resistance to linezolid has occurred but is rare. ${ }^{39} \mathrm{~A}$ more detrimental limitation for selection of this agent is the cost; $\$ 1,677$ for a 10 -day treatment regimen. ${ }^{27}$ Additionally, this option does not have an optimal adverse event profile as a considerable frequency of serious adverse events is observed, eg, thrombocytopenia, peripheral neuropathy, and lactic acidosis. ${ }^{40}$ Daptomycin has also been proven efficacious in cSSSIs but, like other previously mentioned agents, is not active against Gram-negative organisms. ${ }^{41}$ Myopathy may result due to treatment with daptomycin ${ }^{42}$ and, subsequently, alternative treatments may be better options in patients concomitantly on statins. ${ }^{27}$ Tigecycline is approved for the treatment of cSSSIs and has a wide-spectrum of activity including many resistant Gram-positive and Gram-negative pathogens, with the exception of $P$. aeruginosa. ${ }^{43-45}$ Trials comparing tigecycline to vancomycin-aztreonam in patients with cSSSIs revealed that tigecycline has a significantly higher incidence of gastrointestinal events, including nausea and vomiting. ${ }^{46}$ However, most events were mild to moderate and did not lead to discontinuation of therapy. Tigecycline is contraindicated in children and pregnant women as it has a tendency to accumulate in bone $\mathrm{e}^{47,48}$ and cause bone and teeth discoloration. ${ }^{49}$ The above examples describe some available treatment options for cSSSIs. To describe the pharmacokinetic and pharmacodynamic profile of each in full and evaluate the numerous additional therapeutic options, is beyond the scope of this paper and the reader is referred to several reviews. ${ }^{11,12,16,27,38,50,51}$

In view of the above examples, it is evident that there is a need to develop new antimicrobials for the treatment of cSSSIs which have activity against resistant Gram-positive and Gramnegative species with acceptable safety and tolerability. One promising new treatment option is ceftobiprole, a parental cephalosporin antibiotic with a wide-spectrum of activity including several resistant species such as MRSA. This compound has finished phase III trials for the indication cSSSIs but has not yet been FDA approved. This paper reviews the pharmacokinetic/pharmacodynamic (PK/PD) profile of ceftobiprole with respect to its potential role in cSSSIs.

\section{Ceftobiprole pharmacokinetics Plasma concentrations}

Ceftobiprole is available only as a parental agent and is delivered as the water-soluble prodrug ceftobiprole medocaril. It is supplied in single use vials as lyophilized powder corresponding to $500 \mathrm{mg}$ of ceftobiprole, 666.6 $\mathrm{mg}$ of the prodrug, and is reconstituted in water or $5 \%$ dextrose for injection. After reconstitution it is further diluted in $0.9 \%$ sodium chloride, $5 \%$ dextrose, or Lactated Ringer's solution for infusion. The stability and recommended storage conditions of ceftobiprole in each of these solutions varies and should be referenced. ${ }^{52}$ Once infused, the prodrug is converted to the active within seconds by type A esterases. ${ }^{53}$ The protein binding of ceftobiprole has been cited as $16 \%-38 \% .^{53-55}$ Dose proportionality is displayed as clearance, volume of distribution, and dose normalized $\mathrm{AUC}$ and $\mathrm{C}_{\max }$ are consistent in doses ranging from 125-1000 mg. ${ }^{55,56}$ The currently recommended empirical dosing regimen of ceftobiprole for the treatment of cSSSIs is $500 \mathrm{mg}$ every 8 hours as a 2-hour i.v. infusion for 7-14 days. In patients without diabetic foot infection and where gram-positive pathogens only have been documented, the recommended treatment regimen is $500 \mathrm{mg}$ every 12 hours as a 1-hour i.v. infusion for 7-14 days. ${ }^{52}$ Following empirical dosing, the $\mathrm{C}_{\max }$ is $33.0 \mathrm{mg} / \mathrm{L}$ and the $\mathrm{AUC}_{0 \text {-tau }}$ is $102 \mathrm{mg} * \mathrm{hr} / \mathrm{L} \cdot{ }^{52,53,57}$ After a single $500 \mathrm{mg}$ dose as a 1-hour infusion, the $\mathrm{C}_{\max }$ is $34.2 \mathrm{mg} / \mathrm{L}$ and the $\mathrm{AUC}_{0-\infty}$ is $116 \mathrm{mg}{ }^{*} \mathrm{hr} / \mathrm{L} .{ }^{53}$ In a multiple dose study the accumulation of ceftobiprole was found to be negligible with a 12 -hour dosing interval. ${ }^{55}$ Similarly, no significant accumulation occurs during an 8-hour dosing interval. ${ }^{52}$ The pharmacokinetics in patients with cSSSI were similar to healthy volunteers as after dosing $750 \mathrm{mg}$ every 12 hours the $\mathrm{AUC}_{0-\infty}$ was $143 \mathrm{mg} * \mathrm{hr} / \mathrm{L}$ compared to $165 \mathrm{mg} * \mathrm{hr} / \mathrm{L}$, respectively. ${ }^{53}$ In this study the half-life was somewhat lower in patients, 3.06 hours, compared to in healthy volunteers, 4.11 hours. ${ }^{53,58}$ However, in a separate study in healthy volunteers, half-life was reported as 2.61 hours. ${ }^{54}$ It was 
also found that gender does not affect systemic exposure of ceftobiprole if normalized for body weight. ${ }^{53}$

\section{Volume of distribution/tissue distribution}

Ceftobiprole is similar to other beta-lactams in that it distributes primarily to extracellular fluid. The volume of distribution of ceftobiprole is approximately $18 \mathrm{~L}$, reported range of $\mathrm{V}_{\mathrm{ss}} 11 \mathrm{~L}$ to $21.7 \mathrm{~L} .{ }^{56}$ Therefore, it would be assumed that ceftobiprole reaches the site of action to a significant degree, as most bacterial pathogens reside in the extracellular space fluid. To more accurately determine ceftobiprole's concentration at the site of action, a microdialysis study was conducted. In this study, concentrations were measured in subcutaneous soft tissues, ie, adipose tissue and skeletal muscle. Concentrations in these tissues are particularly important to define the PK/PD relationship with regard to cSSSIs. It was found that the AUC ratios of free tissue/free plasma were 0.69 and 0.49 for skeletal muscle and s.c. adipose tissue, respectively, demonstrating that ceftobiprole has the ability to penetrate into these tissues ${ }^{54}$ (Figure 2). Additionally, a study using radiolabeled ceftobiprole was conducted in mice and rats to determine the tissue distribution. It was shown that ceftobiprole distributed rapidly into tissues with the highest tissue/plasma ratio occurring in the kidney, 1:3, and did not penetrate into the brain, tissue/plasma ratio 0:01. ${ }^{52}$ However, the results from radiolabelled studies should be interpreted cautiously as only free drug at the site of action is active and this technique fails to differentiate between central and peripheral compartments.

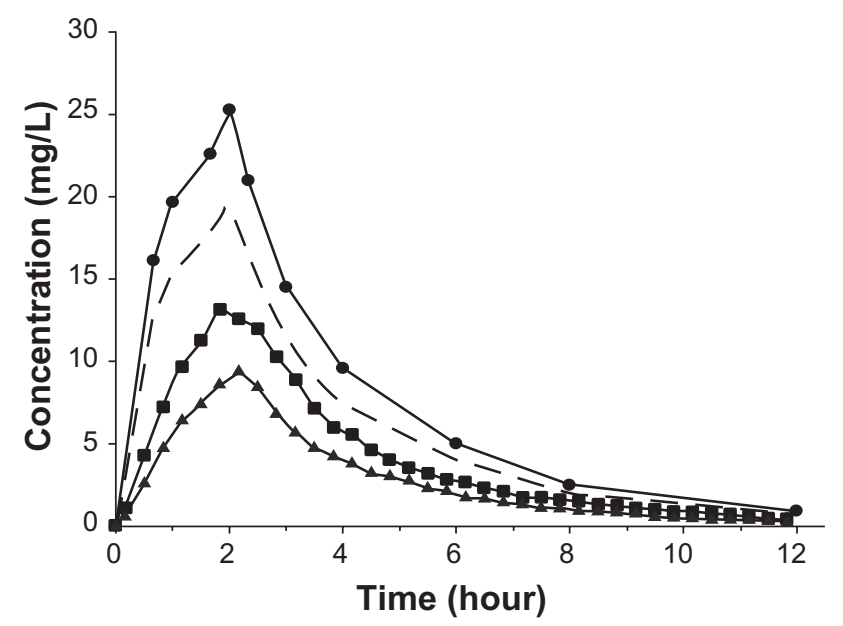

Figure 2 Mean ceftobiprole concentration in plasma (circles), free plasma (dashed line), skeletal muscle (squares), and s.c. adipose tissue (triangles) over 12 h. Reprinted with permission from Barbour A, Schmidt S, Sabarinath SN, et al. Soft-tissue penetration of ceftobiprole in healthy volunteers determined by in vivo microdialysis. Antimicrob Agents Chemother. 2009;53:2773-2776. ${ }^{54}$ Copyright (C) 2009 American Society for Microbiology.

\section{Clearance}

Ceftobiprole is primarily eliminated by glomerular filtration as clearance, range $4.46-5.99 \mathrm{~L} / \mathrm{hr},{ }^{56}$ is highly correlated to creatinine clearance ${ }^{53}$ and approximately equal to the glomerular filtration rate multiplied by the unbound fraction of ceftobiprole. The recovery of unchanged drug in the urine was measured in a single ascending dose study, dose range $125-1000 \mathrm{mg}$ delivered as 0.5 -hour infusions. It was determined that $62 \%$ to $78 \%$ of unchanged drug is recovered in urine of healthy volunteers over 24 hours. ${ }^{55}$ After administration of ceftobiprole, $500 \mathrm{mg} 2 \mathrm{hr}$ infusion $\mathrm{q} 8 \mathrm{hr}$, in healthy volunteers, $\geq 83 \%$ of the drug was found unchanged in the urine, while the primary metabolite, ie, the open-ring metabolite, represented $<7 \% .{ }^{57}$ Ceftobiprole is not thought to be eliminated by active tubular secretion as demonstrated by a probenecid study or metabolism as preclinical and in vitro experiments showed no potential for CYP interactions. ${ }^{53} \mathrm{~A}$ dosing adjustment is recommended in patients with impaired renal function. Patients with a creatinine clearance $\left(\mathrm{Cl}_{\mathrm{cr}}\right)$ of $30<50 \mathrm{~mL} / \mathrm{min}$ should have the dosing interval adjusted to every 12 hours. In patients with a $\mathrm{Cl}_{\text {cr }}<30 \mathrm{~mL} / \mathrm{min}$, the dosing regimen should be $250 \mathrm{mg}$ every 12 hours as a 2 hour i.v. infusion. ${ }^{52}$

\section{Population pharmacokinetics}

Several population PK models have been developed for ceftobiprole. Many of these models were developed for use in Monte Carlo simulations to aid in selection of the optimal dosing regimen. Additionally, these models allow identification of significant covariates and show how these covariates affect the pharmacokinetics and, subsequently, safety and efficacy. In terms of resistance development, theses models can be used to predict the target attainment rate as susceptibility changes, eg, at increased MICs. Phase I data was fit to a two-compartment model to perform Monte Carlo simulations and identify an appropriate dosing regimen for phase II. ${ }^{59}$ Data from phase I and II studies were used to develop a threecompartment model with first-order elimination from the central compartment. ${ }^{60}$ In this model, a hydrolysis compartment was included as the prodrug was infused at a constant rate into this compartment and then hydrolyzed to the active compound. The active drug entered the central compartment via a first-order process. The clearance was dependent on the creatinine clearance in this model. Later the predicted distribution of renal function was used to calculate the target attainment rate and determine the appropriate dosing regimen in patients with various degrees of renal function. Data from phase I, II, and III studies were modeled using a three 
compartment model which included a central compartment and deep and shallow peripheral compartments. In this model, sex, body weight, creatinine clearance, and health status were identified as covariates. ${ }^{61}$ Finally, epithelial lining fluid (ELF) concentrations from healthy volunteers were used to develop a four-compartment model where ELF represented a separate compartment. ${ }^{62}$ This model was more directed at identifying the target concentration at the site of action, ie, ELF, for the indication of nosocomial pneumonia. The application of these models will be discussed in a later section.

\section{Adverse events}

In pivotal phase III trials, ceftobiprole had a safety and tolerability profile similar to competitors. In a study comparing ceftobiprole to vancomycin plus ceftazidime, $56 \%$ of patients in the ceftobiprole arm and $57 \%$ of patients in the comparator arm, experienced at least one adverse event (AE). ${ }^{63}$ Similarly, $7 \%$ of patients in the ceftobiprole arm and $9 \%$ in the competitor arm had at least one serious AE. Four percent of patients from both arms had to discontinue treatment due to an AE. In a study comparing ceftobiprole to vancomycin, only $52 \%$ of patients in the ceftobiprole arm had at least one AE, compared to $51 \%$ in the vancomycin arm. ${ }^{64}$ Four percent of patients in the ceftobiprole arm and 6\% in the vancomycin arm discontinued treatment due to an AE. Six percent of subjects in either arm experienced an AE. Interestingly, dysgeusia was a common $\mathrm{AE}$ in the comparator trial with vancomycin and in trials with healthy volunteers but was not listed in the vancomycin plus ceftazidime trial. The most common AEs for ceftobiprole

Table I Most common adverse events (AEs) in healthy volunteers and patients. ${ }^{55,63,64,66}$

\begin{tabular}{lll}
\hline & Healthy volunteers & Patients \\
\hline Total No. of subjects & 42 & 932 \\
Total No. of subjects & 21 & 507 \\
with at least one AE & & \\
Total No. of subjects with & 0 & 63 \\
at least one serious AE & & \\
Dysgeusia & 17 & 30 \\
Nausea & 10 & 113 \\
Headache & 10 & 68 \\
Abdominal pain & 2 & $*$ \\
Vomiting & 1 & 61 \\
Diarrhea & 1 & 62 \\
Constipation & 0 & 33 \\
Hypersensitivity & 0 & 49 \\
Infusion-site reaction & 1 & 48 \\
\hline
\end{tabular}

ancluding rash and pruritus.

Reprinted with permission from Barbour A, Schmidt S, Rand $\mathrm{KH}$, Derendorf $\mathrm{H}$. Ceftobiprole: a novel cephalosporin with activity against Gram-positive and Gramnegative pathogens, including methicillin-resistant Staphylococcus aureus (MRSA). Int J Antimicrob Agents. 2009;34:1-7. ${ }^{56}$ Copyright (c) 2009 Elsevier. from single and multiple dose studies in healthy volunteers and comparator studies in patients are nausea, headache, dysgeusia, vomiting, diarrhea, constipation, hypersensitivity, and infusion site reaction. Dysgeusia is directly related to ceftobiprole dosing and is thought to be due to the conversion of ceftobiprole medocaril to the active, ceftobiprole, and diacetyl, which is known to produce a caramel-like odor. ${ }^{53,55,65}$ The most common AEs in healthy volunteers and patients with cSSSIs are summarized in Table $1.55,63,64,66$

\section{Ceftobiprole pharmacodynamics}

\section{In vitro}

Ceftobiprole, like other beta-lactams, binds to penicillin binding proteins (PBPs) and inhibits cell-wall synthesis. It has a wide-range of activity against gram-positive pathogens, including MRSA, and a pharmacodynamic profile similar to cefepime and ceftazidime against Gram-negative pathogens. ${ }^{67}$ The in vitro activity of ceftobiprole against isolates collected from cSSSIs during the phase III pivotal studies is presented in Table $2 .{ }^{68}$ One feature of ceftobiprole that makes it a more attractive therapeutic option over many other antibiotics is its activity against several resistant species including MRSA. Ceftobiprole's activity against MRSA is due to its strong affinity for PBP2a (PBP2'), the major cause of resistance to other beta-lactams. ${ }^{69,70}$ Additionally, ceftobiprole has been determined to be primarily bactericidal from in vitro time-kill studies ${ }^{71}$ and by demonstrating $\mathrm{MBC} /$ MIC ratios of $<4$ for a large majority of tested isolates. ${ }^{72}$ Ceftobiprole, however, shows poor affinity for PBP5 and is therefore not active against E. faecium. ${ }^{70}$ Also, ceftobiprole is susceptible to some beta-lactamases which explains the wide range of MICs for P. aeruginosa, Klebsiella pneumo-

Table 2 In vitro susceptibility determined from isolates collected from cSSSIs from phase III trials with ceftobiprole ${ }^{68}$

\begin{tabular}{lll}
\hline & MIC $_{90}(\mathbf{m g} / \mathbf{L})$ & MIC range $(\mathbf{m g} / \mathbf{L})$ \\
\hline MSSA & 0.5 & $0.12-1$ \\
MRSA & 2 & $0.25-4$ \\
Pseudomonas aeruginosa* & $>64$ & $0.25->64$ \\
Enterococcus faecalis & 0.5 & $0.12-0.5$ \\
Enterococcus faecium & $\#$ & $2->64$ \\
Escherichia coli & 0.12 & $0.015->64$ \\
Enterobacter cloacae & $>64$ & $0.03->64$ \\
Klebsiella pneumonia & 32 & $0.03->64$ \\
Streptococcus pyogenes & 0.015 & $0.008-0.25$ \\
Streptococcus agalactiae & 0.12 & $0.015-0.12$ \\
CoNS methicillin susceptible & 0.5 & $0.015-1$ \\
CoNS methicillin-resistant & 2 & $0.06-4$ \\
Proteus mirabilis & 0.12 & $0.015->64$ \\
"Pseudomonas aeruginosa ceftazidime-susceptible MIC $_{90}{ }^{-16 .{ }^{70}}$ & Three isolates collected.
\end{tabular}


nia, and Escherichia coli. For example, $38.7 \%$ of $P$. aeruginosa are inhibited at an $\mathrm{MIC} \leq 1 \mathrm{mg} / \mathrm{L}$, while $85.2 \%$ are inhibited at an $\mathrm{MIC} \leq 8 \mathrm{mg} / \mathrm{L} .{ }^{73}$ Ceftobiprole is resistant to hydrolysis by the TEM-1 and SHV-1 beta-lactamases, similar to the competitors cefepime and ceftazidime. However, it is susceptible to hydrolysis by $P$. aeruginosa displaying high expression of AmpC beta-lactamases or the VIM-2 carbapenemase and E. coli expressing KPC-2 carbapenemase or the CTX-M-15 extended-spectrum beta-lactamase (ESBL). ${ }^{74}$ The $\mathrm{MIC}_{90}$ against Klebsiella species and E. coli for ceftobiprole was $>8 \mathrm{mg} / \mathrm{L}$, while the $\mathrm{MIC}_{90}$ of the carbapenems ertapenem, imipenem, and meropenem were $\leq 0.5 \mathrm{mg} / \mathrm{L}$ in isolates confirmed as ESBL producing. ${ }^{73}$

The post-antibiotic effect (PAE), is a pharmacodynamic measurement that determines if there is any residual effect once the antimicrobial agent has been removed from the system after exposure to suprainhibitory antimicrobial concentrations. For ceftobiprole, PAEs for S. pneumoniae, S. aureus, and E. faecalis were 1.4-3.1 hours, 0-1.7 hours, and 0-0.9 hours, respectively. ${ }^{75}$ Perhaps a more useful in vitro pharmacodynamic measurement is the post-antibiotic sub-MIC effect (PA-SME) as it measures the effect of subinhibitory concentrations after initial exposure to suprainhibitory concentrations. This situation more closely mimics the in vivo situation where there is fluctuation between doses. The PA-SME for $S$. pneumoniae, $S$. aureus, and $E$. faecalis were $4.8>10.3$ hours, $1.5-9.6$ hours, and $3.8>10.7$ hours, respectively. ${ }^{75}$ Comparatively, in vivo PAEs were found to be $0-0.8$ hours and 3.8-4.8 hours for S. pneumonia and $S$. aureus, respectively. ${ }^{76}$

\section{Serial passage studies}

Serial passage studies are often performed with investigational antimicrobials to evaluate the potential for resistance development. When compared to imipenem, ciprofloxacin, and linezolid, ceftobiprole displayed no resistance development using an initial inoculum of $10^{5} \mathrm{CFU} / \mathrm{mL}$ of $S$. aureus (3 MRSA and $1 \mathrm{MSSA}$ ) or against $S$. aureus 745 with an initial inoculum of $2 \times 10^{8} \mathrm{CFU} /$ plate after six to eight passages. ${ }^{70}$ In a separate study, it was shown that after 50 passages resistance to ceftobiprole did not develop in 10 strains of $S$. aureus, including strains with methicillin and/or vancomycin resistance and coagulase negative strains with or without methicillin and/or vancomycin resistance. ${ }^{77}$ The highest observed MIC occurred in the vancomycin-intermediate $S$. aureus (VISA) strain, $8 \mathrm{mg} / \mathrm{L}$, and was four times the original MIC. Similarly, after 50 passages, resistance to ceftobiprole was also not observed in Haemophilus influenzae, six beta-lactamase positive and two negative strains, or Moraxella catarrhalis, two beta-lactamase positive strains. ${ }^{78}$ The highest increase in MIC was four times the original MIC which occurred in one strain of beta-lactamase positive $H$. influenzae, $0.06 \mathrm{mg} / \mathrm{L}$ to $0.25 \mathrm{mg} / \mathrm{L}$. One group was able to demonstrate the occurrence of resistance due to mutations in the $m e c A$ gene by transforming a $m e c A$ negative strain with a plasmid that carries the mutated gene or by the induction of mutations in the mecA transformed strain through serial passages with ceftobiprole. ${ }^{79}$ However, the high inoculum used to induce the mutations after serial passage is not likely to occur in a single patient for a sustained period of time. Surprisingly, in this study, resistance also developed in the mec $A$ negative strain, suggesting a chromosomal mechanism of resistance.

\section{Animal models}

The efficacy of ceftobiprole has also been evaluated in several animal models, the most relevant for the indication of cSSSIs being the mouse abscess model and the murine skin infection model. The peritonitis model will be briefly discussed as peritonitis is often related to, or caused by, surgical site infections, a subclass of cSSSIs. Also noteworthy is the thigh infection model which will be discussed further in the PK/PD section. Animal models which support possible other indications including septicemia, ${ }^{70}$ endocarditis, ${ }^{80}$ and pneumonia/ lung infections ${ }^{81,82}$ will not be discussed here. In the mouse abscess model, mice were inoculated with $S$. aureus I-6, a MRSA strain, or Mu50, a MRSA/VISA strain, under the skin of the groin with abscess developing within 3 days in control animals. ${ }^{70}$ Treatment was administered up to 3 hours after inoculation. Compared to vancomycin $(10 \mathrm{mg} / \mathrm{kg})$ and linezolid $(20 \mathrm{mg} / \mathrm{kg})$, ceftobiprole $(10 \mathrm{mg} / \mathrm{kg})$ treatment resulted in a greater decrease in bacterial counts of MRSA I-6 from untreated controls, 3.42, 0.80, and $5.12 \mathrm{log}$ decreases, respectively. Similarly, with VISA, ceftobiprole $(10 \mathrm{mg} / \mathrm{kg})$ demonstrated a larger decrease in log bacterial counts compared to vancomycin $(40 \mathrm{mg} / \mathrm{kg})$ and linezolid (20 mg/kg), >4.79, 0.13, and 0.64 , respectively, displaying the efficacy of ceftobiprole compared to competitors currently used to treat serious MRSA cSSSIs.

The murine skin infection model compared the activity of ceftobiprole to cefazolin, vancomycin, and linezolid against an MSSA and an MRSA strain in immunocompetent mice. ${ }^{83}$ The activity of ceftobiprole was also compared to cefepime and meropenem-cilastatin against two $P$. aeruginosa strains, an inducible AmpC betalactamase strain (OC4351) and an overproducing AmpC beta-lactamase strain (OC4354), in immunocompromised 
mice. ${ }^{83}$ The mice were inoculated with 6.7-7.0 $\log _{10} \mathrm{CFU} /$ mouse of $S$. aureus or 5.9 to $6.8 \log _{10}$ CFU/mouse of $P$. aeruginosa. After inoculation to the right (MRSA) and left (MSSA) flank, animals were treated with $1.6-100 \mathrm{mg} / \mathrm{kg}$ / day at 1, 3, 25, and 27 hours post infection with ceftobiprole medocaril, cefazolin, vancomycin, or linezolid. One, 2,25 , and 27 hours after inoculation to the left (OC 4351) and right (OC 4354) flanks with $P$. aeruginosa, animals were treated with ceftobiprole medocaril, cefepime, or meropenem-cilastatin with $1.6-100 \mathrm{mg} / \mathrm{kg} / \mathrm{day}$. The reduction of $\mathrm{CFU} / \mathrm{g}$ of infected skin with MSSA and MRSA was greatest with ceftobiprole compared to comparators linezolid and cefazolin across all doses tested. Compared to vancomycin, ceftobiprole treatment resulted in a greater reduction in $\mathrm{CFU} / \mathrm{g}$ for MSSA and MRSA at all doses, except the $100 \mathrm{mg} / \mathrm{kg} /$ day dose at which the decrease in CFU of MRSA/g was equivalent $\left(-1.4 \log _{10}\right.$ change from inoculum). Similarly, ceftobiprole had a greater relative decrease in lesion volume compared to competitors for MSSA and MRSA at all doses, except MSSA with vancomycin at the $100 \mathrm{mg} / \mathrm{kg} /$ day dose, lesion volumes of 555 , 437, 567, and $1181 \mathrm{~mm}^{3}$ for ceftobiprole, vancomycin, linezolid, and untreated controls respectively. Against both $P$. aeruginosa strains, ceftobiprole was as efficacious as meropenem-cilastatin and better than cefepime when comparing change in $\log _{10} \mathrm{CFU} / \mathrm{g}$ at doses of 25 and $100 \mathrm{mg} / \mathrm{kg} / \mathrm{day}$. The $\log _{10}$ change in CFU/g at a dose of $100 \mathrm{mg} / \mathrm{kg} /$ day was $-1.4,1.7,-1.1$ for ceftobiprole, cefepime, and meropenem-cilastatin, respectively, against OC4354. In terms of lesion size, ceftobiprole displayed a greater decrease from untreated controls than cefepime but displayed similar efficacy to meropenem-cilastatin. It should be noted that AmpC levels did not affect ceftobiprole efficacy. This study displayed the potential of ceftobiprole to be used as an empiric agent as it displayed efficacy against two of the most common cSSSI pathogens.

The efficacy of ceftobiprole was also compared to ampicillin, in the mouse Enterococcal peritonitis model. ${ }^{84}$ Infections were induced using an initial inoculum that was $10 \times$ the inoculum that caused death in half the population $\left(\mathrm{LD}_{50}\right)$. The strains used included four E. faecalis strains; one beta-lactamase producing $\left(\mathrm{BLA}^{+}\right)$, two VRE, and one laboratory strain OG1RF. It was found that ceftobiprole displayed similar activity to ampicillin with a $50 \%$ protective dose $\left(\mathrm{PD}_{50}\right)$ for ceftobiprole of $7.7 \mathrm{mg} /$ $\mathrm{kg}, 5.2 \mathrm{mg} / \mathrm{kg}$, and $4.2 \mathrm{mg} / \mathrm{kg}$ compared to $12.5 \mathrm{mg} / \mathrm{kg}$, $16.4 \mathrm{mg} / \mathrm{kg}$, and $8 \mathrm{mg} / \mathrm{kg}$ for ampicillin, against the two VRE strains and the OG1RF strain, respectively. Notably, ceftobiprole displayed a much lower $\mathrm{PD}_{50}$ compared to ampicillin against the $\mathrm{Bla}^{+}$strain, $2 \mathrm{mg} / \mathrm{kg}$ compared to $>100 \mathrm{mg} / \mathrm{kg}$.

\section{Comparator studies}

Two large scale phase III pivotal studies have been conducted in patients with complicated skin and skin structure infections for ceftobiprole. Ceftobiprole was compared to vancomycin in patients with cSSSIs caused by Gram-positive pathogens. ${ }^{64}$ In this study, $500 \mathrm{mg}$ ceftobiprole or $1000 \mathrm{mg}$ vancomycin were administered as 60 minute i.v. infusions ever 12 hours for 7 to 14 days. It was found that ceftobiprole is noninferior to vancomycin with clinical cure rates of $93.3 \%$ and $93.5 \%$, respectively. In a separate study, the efficacy of ceftobiprole was compared to vancomycin plus ceftazidime in patients with cSSSI. In this study, patients in the ceftobiprole arm received $500 \mathrm{mg}$ of ceftobiprole administered as a 120 -minute i.v. infusion every 8 hours plus a 60-minute i.v. infusion of placebo. Patients in the vancomycin plus ceftazidime arm received $1000 \mathrm{mg}$ vancomycin over 60 minutes every 12 hours plus $1000 \mathrm{mg}$ of ceftazidime over 120 minutes every 8 hours. ${ }^{63}$ It was found that ceftobiprole is noninferior to vancomycin plus ceftazidime as the clinical cure rates were $90.5 \%$ and $90.2 \%$, respectively.

While the role of PVL as a virulence factor in MRSA infections is controversial, it is agreed that PVL is associated with community-acquired infections and, as previously mentioned, the increasing frequency of CA-MRSA infections is correlated to a subsequent increase in ED visits. Therefore, it is interesting to note that ceftobiprole had a higher clinical cure rate compared to vancomycin in MRSA PVL-positive infections, 93.1\% (27/29) compared to $84.6 \%(22 / 26)$, although this finding was not statistically significant. ${ }^{64}$ Similarly, ceftobiprole had a higher clinical cure rate for PVL-positive isolates compared to vancomycin plus ceftazidime, $92.1 \%$ (35/38) compared to $84.2 \%$ $(16 / 19){ }^{63}$

\section{Ceftobiprole pharmacokinetics/ pharmacodynamics Establishing pharmacokinetic/ pharmacodynamic targets}

The PK/PD relationship has been established in the mouse thigh and lung infection models. ${ }^{82}$ The more relevant targets for cSSSI would be those established from the thigh infection model and, therefore, the results from these experiments will be the main focus. Dose fractionation studies in neutropenic mice, 
with doses ranging from $0.01 \mathrm{mg} / \mathrm{kg}$ to $160 \mathrm{mg} / \mathrm{kg}$ administered every $3,4,6,8,12$ and/or 24 hours 2 to 10 hours after infection, were used to determine the best correlated PK/PD index and the magnitude of that index resulting in bacterial stasis or a 2-log kill. These studies were performed using 19 bacterial strains including $S$. aureus (MSSA and MRSA), S. pneumoniae (PSSP, PRSP, and one ciprofloxacin-resistant strain), E. coli, K. pneumoniae, Enterobacter cloacae, and P. aeruginosa. From the dose-response curves of the thigh infection model, it was found that the $\mathrm{T}>\mathrm{MIC}$ needed to produce a static effect and $2-\log$ were $19.8 \%$ and $31.9 \%$, respectively, for $S$. aureus, and $15.9 \%$ and $25.1 \%$, respectively, for S. pneumoniae. As typical, a larger magnitude of the PK/PD target was needed to produce stasis and 2-log kill with Gram-negative species, $36.5 \%$ and $54.3 \%$, respectively. These identified targets are in good agreement with the targets currently known to produce efficacy for beta-lactams in both animal models and in the clinic. ${ }^{85-87}$ Additionally, a CART analysis showed that patients with a $\mathrm{T}>\mathrm{MIC}$ of $25 \%$ had a higher probability of therapeutic success, further validating the identified efficacy targets. ${ }^{88}$

\section{Dose selection/evaluation}

The targets identified from dose fractionation studies are often used to predict efficacy using Monte Carlo simulations to find the target attainment rate (TAR). Monte Carlo simulations take variability of the PK, such as inter-patient variability, and PD, ie, the MIC distribution, into account when calculating the TAR. This technique has been used numerous times for the dose selection and dose evaluation for ceftobiprole. In addition to the targets set by dose fractionation experiments, empirical targets are also used as antibiotics in the same class often have approximately the same target. For example, a general PK/PD target for beta-lactams is approximately $40 \% f \mathrm{~T}_{>\mathrm{MIC}}$. However, even among beta-lactam antibiotics the efficacy target varies between subgroups, ie, cephalosporins, penicillins, and carbapenems, and specific pathogens. ${ }^{85-87}$ Monte Carlo simulations were performed first using PK data from 12 healthy volunteers, where the TAR for the PK/PD targets of $f \mathrm{~T}_{>\text {MIC }} 30 \%-60 \%$ were calculated for numerous doses, 250-1000 mg administered every 12 or 8 hours. ${ }^{59}$ From these simulations it was concluded that a $750 \mathrm{mg}$ dose given every 12 hours had a high probability of efficacy for organisms with MICs up to $4 \mathrm{mg} / \mathrm{L}$. Monte Carlo simulations were later performed using a larger and more diverse patient population. ${ }^{89}$ In these simulations, the PK was characterized using data from 150 subjects from both phase I and phase II trials. Doses of $500 \mathrm{mg}$ every 8 hours delivered as 0.5-, 1-, or 2-hour i.v. infusions and $500 \mathrm{mg}$ every 12 delivered as a 1-hour i.v. infusion were simulated to determine the TAR for targets of $30 \%-60 \% f \mathrm{~T}_{>\mathrm{MIC}}$. Additionally, the TAR was calculated in patients with renal impairment, $\mathrm{Cl}_{\mathrm{cr}}=30 \mathrm{~mL} / \mathrm{min}$ dosed with $250 \mathrm{mg}$ every 8 hours as a 2-hour i.v. infusion, and $\mathrm{Cl}_{\mathrm{cr}}=50 \mathrm{~mL} / \mathrm{min}$ dosed with $500 \mathrm{mg}$ every 12 hours as a 2 -hour i.v. infusion. Based on these simulations, $500 \mathrm{mg}$ administered as a 1-hour i.v. infusion every 12 hours was recommended for the indication of cSSSIs. Also, a dosing regimen of $500 \mathrm{mg}$ as a 2-hour i.v. infusion every 8 hours was deemed an appropriate dose in patients with mild to moderate renal impairment, ie, $\mathrm{Cl}_{\mathrm{cr}} \sim 50 \mathrm{~mL} / \mathrm{min}$. Finally, simulations were done using a model that was developed using data from phase I, II, and III trials and the individual patient demographics from patients in the phase III trials using a dosing regimen of $500 \mathrm{mg}$ every 8 hours as a 2-hour i.v. infusion. ${ }^{61}$ It was found that the overall TAR for targets of $f \mathrm{~T}_{>\mathrm{MIC}} 30 \%$ and $50 \%$ were $94.6 \%$ and $93.7 \%$, respectively, based on the MIC distribution and a creatinine clearance of $\geq 80 \mathrm{~mL} / \mathrm{min} .{ }^{88}$ The dose of $500 \mathrm{mg}$ delivered as a 2-hour infusion was also validated in the microdialysis study where it was found that concentrations in the extracellular fluid of s.c. adipose tissue and skeletal muscle met the PK/PD target of $f \mathrm{~T}_{>\mathrm{MIC}} 40 \%$ for organisms with a MIC $\leq 2 \mathrm{mg} / \mathrm{L}^{54}$

\section{Discussion}

In North America, $44.6 \%$ of SSTIs are caused by S. aureus, of which $35.9 \%$ are methicillin-resistant. ${ }^{17}$ The second most commonly identified pathogen, $P$. aeruginosa, accounts for $11.1 \%$ of SSTIs, while the third and fourth most commonly isolated pathogens were Enterococcus species (9.3\%) and E. coli (7.2\%), respectively. Additionally, cSSSIs can be polymicrobial and include both Gram-positive and Gram-negative pathogens, eg, diabetic foot infections. Therefore, due to the wide-spectrum of causative pathogens and various resistance mechanisms, an obvious challenge arises in identifying a first-line single agent or even combination therapy for empiric treatment prior to microbial identification. Based on its activity profile, ceftobiprole has the potential to serve as a single agent for the empiric treatment of cSSSIs. However, pathogen identification and characterization is still needed as ceftobiprole is susceptible to some beta-lactamases. For example, while activity is observed against $E$. coli, $\mathrm{MIC}_{90} 0.12 \mathrm{mg} / \mathrm{L}$, in species producing ESBLs $(\sim 6 \%),{ }^{17,67}$ the $\mathrm{MIC}_{90}$ is reported as $>8 \mathrm{mg} / \mathrm{L} .{ }^{67}$ Additionally, ceftobiprole does not display good activity against E. faecium. ${ }^{70}$ Another minor limitation of ceftobiprole, is that the mechanism of action does not inhibit protein synthesis as some competitors. Down regulating PVL, a possible virulence factor, could be advantageous in CA-MRSA infections. ${ }^{27}$

In conclusion, ceftobiprole has several characteristics that make it a promising single agent for use empirically to treat cSSSIs including its spectrum of activity, 
distributional properties, and excellent safety and tolerability profile.

\section{Disclosure}

Dr Derendorf has previously received funding from Johnson and Johnson Pharmaceutical Research and Development.

\section{References}

1. Ibrahim EH, Sherman G, Ward S, Fraser VJ, Kollef MH. The influence of inadequate antimicrobial treatment of bloodstream infections on patient outcomes in the ICU setting. Chest. 2000;118:146-155.

2. Iregui M, Ward S, Sherman G, Fraser VJ, Kollef MH. Clinical importance of delays in the initiation of appropriate antibiotic treatment for ventilator-associated pneumonia. Chest. 2002;122:262-268.

3. Eagye KJ, Kim A, Laohavaleeson S, Kuti JL, Nicolau DP. Surgical site infections: does inadequate antibiotic therapy affect patient outcomes? Surg Infect (Larchmt). 2009;10:323-331.

4. Itani KM, Akhras KS, Stellhorn R, et al. Outcomes associated with initial versus later vancomycin use in patients with complicated skin and skin-structure infections. Pharmacoeconomics. 2009;27:421-430.

5. Cosgrove SE, QiY, Kaye KS, et al. The impact of methicillin resistance in Staphyblococcus aureus bacteremia on patient outcomes: mortality, length of stay, and hospital charges. Infect Control Hosp Epidemiol. 2005;26:166-174.

6. Lodise TP, McKinnon PS. Clinical and economic impact of methicillin resistance in patients with Staphylococcus aureus bacteremia. Diagn Microbiol Infect Dis. 2005;52:113-122.

7. Falagas ME, Barefoot L, Griffith J, Ruthazar R, Snydman DR. Risk factors leading to clinical failure in the treatment of intra-abdominal or skin/soft tissue infections. Eur J Clin Microbiol Infect Dis. 1996;15:9 13-921.

8. Zilberberg MD, Shorr AF, Micek ST, et al. Epidemiology and outcomes of hospitalizations with complicated skin and skin-structure infections: implications of healthcare-associated infection risk factors. Infect Control Hosp Epidemiol. 2009;30:1203-1210.

9. Ruhe JJ, Smith N, Bradsher RW, Menon A. Community-onset methicillin-resistant Staphylococcus aureus skin and soft-tissue infections: impact of antimicrobial therapy on outcome. Clin Infect Dis. 2007;44:777-784.

10. Research C-CfDEa. Guidance for Industry: Uncomplicated and Complicated Skin and Skin Structure Infections - Developing Antimicrobial Drugs for Treatment. In: Administration USDoHaHSFaD (ed.). Rockville, MD 1998.

11. Nichols RL. Optimal treatment of complicated skin and skin structure infections. J Antimicrob Chemother. 1999;44 Suppl A:19-23.

12. Lee SY, Kuti JL, Nicolau DP. Antimicrobial management of complicated skin and skin structure infections in the era of emerging resistance. Surg Infect (Larchmt). 2005;6:283-295.

13. DiNubile MJ, Lipsky BA. Complicated infections of skin and skin structures: when the infection is more than skin deep. J Antimicrob Chemother. 2004;53 Suppl 2:ii37-ii50.

14. Lee MC, Rios AM, Aten MF, et al. Management and outcome of children with skin and soft tissue abscesses caused by community-acquired methicillin-resistant Staphylococcus aureus. Pediatr Infect Dis J. 2004;23:123-127.

15. Llera JL, Levy RC. Treatment of cutaneous abscess: a double-blind clinical study. Ann Emerg Med. 1985;14:15-19.

16. Fung HB, Chang JY, Kuczynski S. A practical guide to the treatment of complicated skin and soft tissue infections. Drugs. 2003;63: 1459-1480.

17. Moet GJ, Jones RN, Biedenbach DJ, Stilwell MG, Fritsche TR. Contemporary causes of skin and soft tissue infections in North America, Latin America, and Europe: report from the SENTRY Antimicrobial Surveillance Program (1998-2004). Diagn Microbiol Infect Dis. 2007;57:7-13.
18. Klevens RM, Edwards JR, Richards CL Jr, et al. Estimating health care-associated infections and deaths in U.S. hospitals, 2002. Public Health Rep. 2007;122:160-166.

19. Vandenesch F, Naimi T, Enright MC, et al. Community-acquired methicillin-resistant Staphylococcus aureus carrying Panton-Valentine leukocidin genes: worldwide emergence. Emerg Infect Dis. 2003;9:978-984.

20. King MD, Humphrey BJ, Wang YF, et al. Emergence of communityacquired methicillin-resistant Staphylococcus aureus USA 300 clone as the predominant cause of skin and soft-tissue infections. Ann Intern Med. 2006;144:309-317.

21. Tillotson GS, Draghi DC, Sahm DF, et al. Susceptibility of Staphylococcus aureus isolated from skin and wound infections in the United States 2005-07: laboratory-based surveillance study. JAntimicrob Chemother. 2008;62:109-115.

22. Pallin DJ, Egan DJ, Pelletier AJ, et al. Increased US emergency department visits for skin and soft tissue infections, and changes in antibiotic choices, during the emergence of community-associated methicillin-resistant Staphylococcus aureus. Ann Emerg Med. 2008;51:291-298.

23. McCaig LF, McDonald LC, Mandal S, Jernigan DB. Staphylococcus aureus-associated skin and soft tissue infections in ambulatory care. Emerg Infect Dis. 2006;12:1715-1723.

24. Hersh AL, Chambers HF, Maselli JH, Gonzales R. National trends in ambulatory visits and antibiotic prescribing for skin and soft-tissue infections. Arch Int Med. 2008;168:1585-1591.

25. Gupta K, Macintyre A, Vanasse G, Dembry LM. Trends in prescribing beta-lactam antibiotics for treatment of community-associated methicillin-resistant Staphylococcus aureus infections. J Clin Microbiol. 2007;45:3930-3934.

26. Gorwitz RJ, Jernigan DB, Powers JH, Jernigan JA, Participants. Startegies for clinical management of MRSA in the community: summary of an expert's mettings convened by the CDC. In: Prevention CfDCa (ed.). Atlanta, GA 2005.

27. Powell JP, Wenzel RP. Antibiotic options for treating communityacquired MRSA. Expert Rev Anti Infect Ther. 2008;6:299-307.

28. Sader HS, Jones RN. Antimicrobial susceptibility of Gram-positive bacteria isolated from US medical centers: results of the Daptomycin Surveillance Program (2007-2008). Diagn Microbiol Infect Dis. 2009; $65: 158-162$.

29. Prevention CfDCa. Staphylococcus aureus resistant to vancomycinUnited States, 2002. MMWR Morb Mortal Wkly Rep. 2002; 51:565-567.

30. Prevention CfDCa. Vancomycin-resistant Staphylococcus aureusPennsylvania, 2002. MMWR Morb Mortal Wkly Rep. 2002;51:902.

31. Prevention CfDCa. Vancomycin-resistant Staphylococcus aureus-New York, 2004. MMWR Morb Mortal Wkly Rep. 2004;53:322-323.

32. DSM Pharmaceuticals I. Synercid I.V. Prescribing Information. Greenville, NC 2003.

33. Drew RH, Perfect JR, Srinath L, et al. Treatment of methicillin-resistant staphylococcus aureus infections with quinupristin-dalfopristin in patients intolerant of or failing prior therapy. For the Synercid Emergency-Use Study Group. J Antimicrob Chemother. 2000;46:775-784.

34. Nichols RL, Graham DR, Barriere SL, et al. Treatment of hospitalized patients with complicated gram-positive skin and skin structure infections: two randomized, multicentre studies of quinupristin/dalfopristin versus cefazolin, oxacillin or vancomycin. Synercid Skin and Skin Structure Infection Group. J Antimicrob Chemother. 1999;44:263-273.

35. Ling TK, Fung KS, Cheng AF. In vitro activity and post-antibiotic effect of quinupristin/dalfopristin (Synercid). Chemotherapy. 2001;47: 243-249.

36. Auckenthaler R, Courvalin P, Feger C, Roche G. In vitro activity of quinupristin/dalfopristin in comparison with five antibiotics against worldwide clinical isolates of staphylococci. Clin Microbiol Infect. 2000;6:608-612.

37. Fritsche TR, Kirby JT, Jones RN. In vitro activity of tigecycline (GAR-936) tested against 11,859 recent clinical isolates associated with community-acquired respiratory tract and gram-positive cutaneous infections. Diag Microbiol Infect Dis. 2004;49:201-209. 
38. Paterson DL. Clinical experience with recently approved antibiotics. Curr Opin Pharmacol. 2006;6:486-490.

39. Farrell DJ, Mendes RE, Ross JE, Jones RN. Linezolid surveillance program results for 2008 (LEADER Program for 2008). Diag Microbio Infect Dis. 2009;65:392-403.

40. Beekmann SE, Gilbert DN, Polgreen PM. Toxicity of extended courses of linezolid: results of an Infectious Diseases Society of America Emerging Infections Network survey. Diag Microbiol Infect Dis. 2008;62:407-410.

41. Arbeit RD, Maki D, Tally FP, Campanaro E, Eisenstein BI. The safety and efficacy of daptomycin for the treatment of complicated skin and skin-structure infections. Clin Infect Dis. 2004;38:1673-1681.

42. Oleson FB Jr, Berman CL, Kirkpatrick JB, et al. Once-daily dosing in dogs optimizes daptomycin safety. Antimicrob Agents Chemother. 2000;44:2948-2953.

43. Petersen PJ, Jacobus NV, Weiss WJ, Sum PE, Testa RT. In vitro and in vivo antibacterial activities of a novel glycylcycline, the 9-t-butylglycylamido derivative of minocycline (GAR-936). Antimicrob Agents Chemother. 1999;43:738-744.

44. Dowzicky MJ, Park CH. Update on antimicrobial susceptibility rates among gram-negative and gram-positive organisms in the United States: results from the Tigecycline Evaluation and Surveillance Trial (TEST) 2005 to 2007. Clin Ther. 2008;30:2040-2050.

45. Papaparaskevas J, Tzouvelekis LS, Tsakris A, Pittaras TE, Legakis NJ. In vitro activity of tigecycline against 2423 clinical isolates and comparison of the available interpretation breakpoints. Diagn Microbiol Infect Dis. 2010;66:187-194.

46. Ellis-Grosse EJ, Babinchak T, Dartois N, Rose G, Loh E. The efficacy and safety of tigecycline in the treatment of skin and skin-structure infections: results of 2 double-blind phase 3 comparison studies with vancomycin-aztreonam. Clin Infect Dis. 2005;41 Suppl 5:S341-S353.

47. Rodvold KA, Gotfried MH, Cwik M, et al. Serum, tissue and body fluid concentrations of tigecycline after a single $100 \mathrm{mg}$ dose. J Antimicrob Chemother. 2006;58:1221-1229.

48. Tombs N. Tissue distribution of GAR-936 a broad-spectrum antibiotic, in male rats. Interscience Conference on Antimicrobial Agents and Chemotherapy. San Francisco 1999.

49. Wyeth Pharmaceuticals I. Tygacil Prescribing Information. Philadelphia 2009.

50. Stevens DL, Bisno AL, Chambers HF, et al. Practice guidelines for the diagnosis and management of skin and soft-tissue infections. Clin Infect Dis. 2005;41:1373-1406.

51. Nathwani D. New antibiotics for the management of complicated skin and soft tissue infections: are they any better? Int J Antimicrob Agents. 2009;34 Suppl 1:S24-S29.

52. Janssen-Ortho I. Zeftera Prescribing Information. Vol. 2008. Toronto, Ontario: Janssen-Ortho Inc. 2008.

53. Murthy B, Schmitt-Hoffmann A. Pharmacokinetics and pharmacodynamics of ceftobiprole, an anti-MRSA cephalosporin with broadspectrum activity. Clin Pharmacokinet. 2008;47:21-33.

54. Barbour A, Schmidt S, Sabarinath SN, et al. Soft-tissue penetration of ceftobiprole in healthy volunteers determined by in vivo microdialysis. Antimicrob Agents Chemother. 2009;53:2773-2776.

55. Schmitt-Hoffmann A, Roos B, Schleimer M, et al. Single-dose pharmacokinetics and safety of a novel broad-spectrum cephalosporin (BAL5788) in healthy volunteers. Antimicrob Agents Chemother. 2004;48:2570-2575.

56. Barbour A, Schmidt S, Rand KH, Derendorf H. Ceftobiprole: a novel cephalosporin with activity against Gram-positive and Gram-negative pathogens, including methicillin-resistant Staphylococcus aureus (MRSA). Int J Antimicrob Agents. 2009;34:1-7.

57. Murthy BSD, Wexler D, Balis D, Chang I, Desai-Kreiger D, Noel G. Pharmacokinetics of ceftobiprole following single and multiple intravenous infusions administered to healthy subjects. 17th European Congress of Clinical Microbiology and Infectious Diseases Munich, Germany 2007.
58. Schmitt-Hoffmann AHM, Heep M, et al. BAL5788 in patients with complicated skin and skin structure infections caused by Gram-positive pathogens including methicillin-resistant Staphylococcus species (MRSS). 14th European Congress of Clinical Microbiology and Infectious Diseases. Prague 2004.

59. Mouton JW, Schmitt-Hoffmann A, Shapiro S, Nashed N, Punt NC. Use of Monte Carlo simulations to select therapeutic doses and provisional breakpoints of BAL9141. Antimicrob Agents Chemother. 2004;48:1713-1718.

60. Lodise TP Jr, Pypstra R, Kahn JB, et al. Probability of target attainment for ceftobiprole as derived from a population pharmacokinetic analysis of 150 subjects. Antimicrob Agents Chemother. 2007;51:2378-2387.

61. Kimko H, Murthy B, Xu X, et al. Population pharmacokinetic analysis of ceftobiprole for treatment of complicated skin and skin structure infections. Antimicrob Agents Chemother. 2009;53:1228-1230.

62. Rodvold KA, Nicolau DP, Lodise TP, et al. Identifying exposure targets for treatment of staphylococcal pneumonia with ceftobiprole. Antimicrob Agents Chemother. 2009;53:3294-3301.

63. Noel GJ, Bush K, Bagchi P, Ianus J, Strauss RS. A randomized, double-blind trial comparing ceftobiprole medocaril with vancomycin plus ceftazidime for the treatment of patients with complicated skin and skin-structure infections. Clin Infect Dis. 2008;46:647-655.

64. Noel GJ, Strauss RS, Amsler K, et al. Results of a double-blind, randomized trial of ceftobiprole treatment of complicated skin and skin structure infections caused by gram-positive bacteria. Antimicrob Agents Chemother. 2008;52:37-44.

65. Chew TA, Smith JM. Detection of diacetyl (caramel odor) in presumptive identification of the "Streptococcus milleri" group. J Clin Microbiol. 1992;30:3028-3029.

66. Schmitt-Hoffmann A, Nyman L, Roos B, et al. Multiple-dose pharmacokinetics and safety of a novel broad-spectrum cephalosporin (BAL5788) in healthy volunteers. Antimicrob Agents Chemother. 2004;48:2576-2580.

67. Fritsche TR, Sader HS, Jones RN. Antimicrobial activity of ceftobiprole, a novel anti-methicillin-resistant Staphylococcus aureus cephalosporin, tested against contemporary pathogens: results from the SENTRY Antimicrobial Surveillance Program (2005-2006). Diagn Microbiol Infect Dis. 2008;61:86-95.

68. Amsler KM, Davies TA, Shang W, Jacobs MR, Bush K. In vitro activity of ceftobiprole against pathogens from two phase 3 clinical trials of complicated skin and skin structure infections. Antimicrob Agents Chemother. 2008;52:3418-3423.

69. Davies TA, Page MG, Shang W, et al. Binding of ceftobiprole and comparators to the penicillin-binding proteins of Escherichia coli, Pseudomonas aeruginosa, Staphylococcus aureus, and Streptococcus pneumoniae. Antimicrob Agents Chemother. 2007;51: 2621-2624

70. Hebeisen P, Heinze-Krauss I, Angehrn P, et al. In vitro and in vivo properties of Ro 63-9141, a novel broad-spectrum cephalosporin with activity against methicillin-resistant staphylococci. Antimicrob Agents Chemother. 2001;45:825-836.

71. Deshpande LM, Jones RN. Bactericidal activity and synergy studies of BAL9141, a novel pyrrolidinone-3-ylidenemethyl cephem, tested against streptococci, enterococci and methicillin-resistant staphylococci. Clin Microbiol Infect. 2003;9:1120-1124.

72. Deshpande L, Rhomberg PR, Fritsche TR, Sader HS, Jones RN. Bactericidal activity of BAL9141, a novel parenteral cephalosporin against contemporary Gram-positive and Gram-negative isolates. Diag Microbiol Infect Dis. 2004;50:73-75.

73. Fritsche TR, Sader HS, Jones RN. Potency and spectrum of garenoxacin tested against an international collection of skin and soft tissue infection pathogens: report from the SENTRY antimicrobial surveillance program (1999-2004). Diagn Microbiol Infect Dis. 2007;58:19-26. 
74. Queenan AM, Shang W, Kania M, Page MG, Bush K. Interactions of ceftobiprole with beta-lactamases from molecular classes A to D. Antimicrob Agents Chemother. 2007;51:3089-3095.

75. Pankuch GA, Appelbaum PC. Postantibiotic effect of ceftobiprole against 12 Gram-positive organisms. Antimicrob Agents Chemother. 2006;50:3956-3958

76. Andes D WC. In-vivo pharmcodynamics of RO 63-9141 against multiple bacterial pathogens 40th Interscience Conference on Antimicrobial Agents and Chemotherapy. Toronto 2000.

77. Bogdanovich T, Ednie LM, Shapiro S, Appelbaum PC. Antistaphylococcal activity of ceftobiprole, a new broad-spectrum cephalosporin. Antimicrob Agents Chemother. 2005;49:4210-4219.

78. Bogdanovich T, Clark C, Ednie L, et al. Activities of ceftobiprole, a novel broad-spectrum cephalosporin, against Haemophilus influenzae and Moraxella catarrhalis. Antimicrob Agents Chemother. 2006;50:2050-2057.

79. Banerjee R, Gretes M, Basuino L, Strynadka N, Chambers HF. In vitro selection and characterization of ceftobiprole-resistant methicillinresistant Staphylococcus aureus. Antimicrob Agents Chemother. 2008;52:2089-2096.

80. Chambers HF. Evaluation of ceftobiprole in a rabbit model of aortic valve endocarditis due to methicillin-resistant and vancomycinintermediate Staphylococcus aureus. Antimicrob Agents Chemother. 2005;49:884-888.

81. Azoulay-Dupuis E, Bedos JP, Mohler J, et al. Efficacy of BAL5788, a prodrug of cephalosporin BAL9141, in a mouse model of acute pneumococcal pneumonia. Antimicrob Agents Chemother. 2004;48: 1105-1111.
82. Craig WA, Andes DR. In vivo pharmacodynamics of ceftobiprole against multiple bacterial pathogens in murine thigh and lung infection models. Antimicrob Agents Chemother. 2008;52:3492-3496.

83. Fernandez J, Hilliard JJ, Abbanat D, et al. In vivo activity of ceftobiprole in murine skin infections due to Staphylococcus aureus and Pseudomonas aeruginosa. Antimicrob Agents Chemother. 2010;54:116-125.

84. Arias CA, Singh KV, Panesso D, Murray BE. Evaluation of ceftobiprole medocaril against Enterococcus faecalis in a mouse peritonitis model. J Antimicrob Chemother. 2007;60:594-598.

85. Craig WA. Pharmacokinetic/pharmacodynamic parameters: rationale for antibacterial dosing of mice and men. Clin Infect Dis. 1998;26:1-10; quiz 11-12.

86. Burgess DS, Frei CR. Comparison of beta-lactam regimens for the treatment of gram-negative pulmonary infections in the intensive care unit based on pharmacokinetics/pharmacodynamics. J Antimicrob Chemother. 2005;56:893-898.

87. Bhavnani SM. WHICH PK-PD MEASURE FOR WHICH DRUG? International Society of Anti-Infective Pharmacology: WA Craig Symposium. Madison, WI, USA 2008.

88. Kimko H, Xu X, Nandy $\mathrm{P}$, et al. Pharmacodynamic profiling of ceftobiprole for treatment of complicated skin and skin structure infections. Antimicrob Agents Chemother. 2009;53:3371-3374.

89. Lodise TPJ, Pypstra R, Kahn JB, et al. Probability of target attainment for ceftobiprole as derived from a population pharmacokinetic analysis of 150 subjects. Antimicrob Agents Chemother. 2007;51:2378-2387.
Therapeutics and Clinical Risk Management

\section{Publish your work in this journal}

Therapeutics and Clinical Risk Management is an international, peerreviewed journal of clinical therapeutics and risk management, focusing on concise rapid reporting of clinical studies in all therapeutic areas, outcomes, safety, and programs for the effective, safe, and sustained use of medicines. This journal is indexed on PubMed Central, CAS,

\section{Dovepress}

EMBase, Scopus and the Elsevier Bibliographic databases. The manuscript management system is completely online and includes a very quick and fair peer-review system, which is all easy to use. Visit http://www.dovepress.com/testimonials.php to read real quotes from published authors.

Submit your manuscript here: http://www.dovepress.com/therapeutics-and-clinical-risk-management-journal 